\title{
Systematic Review \\ Efficacy of Instruments for Professional Oral Hygiene on Dental Implants: A Systematic Review
}

\author{
Domenico Baldi *, Luisa De Giorgis *, Maria Menini ${ }^{D}$, Franco Motta and Jacopo Colombo * \\ Department of Surgical Sciences (DISC), Division of Prosthetic Dentistry, University of Genoa, Largo R. Benzi 10, \\ 16132 Genoa, Italy; maria.menini@unige.it (M.M.); motta.studio@tiscali.it (F.M.) \\ * Correspondence: baldi.domenico@unige.it (D.B.); ludeg94@gmail.com (L.D.G.); jacopocolo@tiscali.it (J.C.); \\ Tel.: +39-0187525931 (D.B.); +39-3488936274 (L.D.G.); +39-0187525931 or +39-3498504815 (J.C.)
}

Citation: Baldi, D.; De Giorgis, L.; Menini, M.; Motta, F.; Colombo, J. Efficacy of Instruments for Professional Oral Hygiene on Dental Implants: A Systematic Review. Appl. Sci. 2022, 12, 26. https://doi.org/ 10.3390/app12010026

Academic Editors: Luca Testarelli and Giuliana Muzio

Received: 29 June 2021

Accepted: 11 August 2021

Published: 21 December 2021

Publisher's Note: MDPI stays neutral with regard to jurisdictional claims in published maps and institutional affiliations.

Copyright: (c) 2021 by the authors. Licensee MDPI, Basel, Switzerland. This article is an open access article distributed under the terms and conditions of the Creative Commons Attribution (CC BY) license (https:// creativecommons.org/licenses/by/ $4.0 /)$.

\begin{abstract}
Professional oral hygiene is fundamental to prevent peri-implant disease. Appropriate instruments should be used in patients with restorations supported by dental implants: they should be effective in deposits removal without damaging the implant components surface. The aim of the present study is to investigate and summarize the results regarding the efficacy of oral hygiene techniques described in the literature in the last 10 years in patients rehabilitated with dental implants not affected by perimplantitis. The present systematic review was conducted according to guidelines reported in the indications of the Preferred Reporting Items for Systematic Review and Meta-Analysis (PRISMA). The focused question was: "Which are the most effective instruments for professional oral hygiene on implants not affected by perimplantitis?". The initial database search yielded a total of 934 entries found in PubMed ${ }^{\circledR} /$ MEDLINE and Cochrane Library. After full text review and application of the eligibility criteria, the final selection consisted of 19 articles. The risk of bias of included studies was assessed using the Newcastle Ottawa scale (NOS) and the Cochrane Handbook for Systematic Reviews of Interventions. Curette, scalers and air polishing were the devices most frequently investigated in the included studies. In particular, glycine powder air polishing appeared to be significantly effective in reducing peri-implant inflammation and plaque around implants. The application of the more recent erythritol powder air polishing also yielded good clinical outcomes. Further studies are needed to improve the knowledge on the topic in order to develop standardized protocols and understand the specific indications for different types of implant-supported rehabilitations.
\end{abstract}

Keywords: dental implants; oral hygiene techniques; curette; ultrasonic scaler; air polishing; air flow; glycine; sodium bicarbonate; erythritol

\section{Introduction}

Professional oral hygiene, in synergy with daily home oral hygiene, strongly contributes to the prevention of peri-implant disease. It is aimed at maintaining the health of oral tissues by removing plaque and tartar that accumulate over teeth and restorations. The frequency of follow-up appointments is established by the dentist or the dental hygienist on the basis of oral condition, the characteristics of the patient and his/her ability to maintain a good oral hygiene.

Different instruments and techniques might be used in a single oral hygiene session: tartar can be fragmented and removed by the scaler which generates high-frequency vibrations. Ultrasonic devices have several dental applications especially in dental hygiene, surgery and prosthodontic [1-3]. The advantages of ultrasonic instruments include: precision, preservation of soft tissues and reduction of operating times. However, metal ultrasonic instruments have been reported to damage implant and prosthodontic surfaces while plastic tips might be less effective in deposits removal [4]. 
An air polishing treatment can be performed which uses a jet of air, water and microparticles, the most common being sodium bicarbonate, glycine and erythritol, in order to remove stains on the surface of the teeth [5-7].

Manual instruments such as curettes are also available, made by different possible materials (metal alloy or plastic materials). The dentist or the dental hygienist can use metal curettes to deeply remove tartar and carefully clean the root of natural teeth, while their use has been reported to damage the surface of implant prosthodontic components. For this reason, plastic instruments might be preferred when instrumenting dental implants. However, they are more fragile and their bulky design makes it difficult to reach all the surfaces to be cleaned.

The dental implant is a predictable replacement for natural teeth and it requires constant maintenance and monitoring for long-term success [8]. Appropriate instruments should be used in patients with prosthetic restorations supported by implants in order to accurately clean them without damaging their surfaces. Metallic tools could scratch the titanium surface, creating a rougher surface that might favour microbic adhesion and plaque build-up. It is widely accepted that local debridement of implants should be performed with titanium instruments or instruments that are softer than titanium $[9,10]$. Tools such as plastic curettes or air polishing might be preferrable [11]. However, their efficacy and effectiveness has been questioned and plastic remnants could remain after instrumentation [12]. In addition, alterations of the implant surface could be caused by air polishing with large-sized powders with higher cleaning capacity [13].

The aim of the present systematic review is to investigate and summarize data available in the literature on the efficacy of instruments for professional oral hygiene of dental implants described in the literature in the last 10 years in patients not affected by perimplantitis.

\section{Materials and Methods}

The present systematic review was conducted according to guidelines reported in the indications of the Preferred Reporting Items for Systematic Review and Meta-Analysis (PRISMA) [14].

The Prospero ID is CRD42021275812.

The focused question was: Which are the most effective instruments for professional oral hygiene of dental implants not affected by perimplantitis?

The focused question was established according to PICO strategy:

- $\quad$ Population: patients with dental implants not affected by perimplantitis

- Intervention: any instrument for professional oral hygiene on dental implants

- Comparison: any instrument for professional oral hygiene on dental implants different from intervention

- $\quad$ Outcomes: cleaning efficacy

\subsection{Search Strategy}

The National Library of Medicine (PubMed ${ }^{\circledR} /$ MEDLINE) and Cochrane Library were used as the Internet sources to search for papers that satisfied the study purpose.

The last search was performed on the 21 April 2021. We used the Mesh term "dental implants" combined using the boolean operator AND with the following search terms: "oral hygiene", "curette", "ultrasonic scaler", "air polishing", " air flow", "glycine", "sodium bicarbonate", "erythritol".

All the original studies investigating professional oral hygiene techniques were included if they met the following inclusion criteria:

- $\quad$ oral hygiene techniques on dental implants or dental implant materials,

- $\quad$ studies of the last ten years (since 1 January 2010),

- no patients affected by perimplantitis

- $\quad$ no patients with orthodontic appliances

- no patients affected by systemic pathologies 
Eligible articles included: comparative studies, RCT, cohort studies, case control studies, in vitro comparative studies, animal comparative studies, clinical trials, study in vitro. Restrictions in terms of language were applied: only papers written in Italian or English language were included. No publication status restrictions were imposed. Non original studies (i.e., narrative or systematic reviews, editorials, expert opinions etc.) were excluded. However, full texts of narrative and systematic reviews dealing with the topic of the present review were obtained in order to screen their reference list for possible additional studies to be included. Similarly, a hand search was performed by screening the reference list of all included publications to select potentially relevant additional studies. Redundant studies were excluded.

\subsection{Screening and Selection}

Titles and abstracts of the searches were screened by two independent reviewers (J.C., L.D.G.) for possible inclusion. The full texts of all studies of possible relevance were then obtained for independent assessment by the reviewers. Disagreements between reviewers were resolved by discussion between the two review authors; if no agreement could be reached, a third author decided (D.B.).

When we did not manage to download the full text version, the corresponding author was contacted.

\subsection{Data Extraction}

The following data were extracted: author(s), publication year, title of the paper, study design, surface treated/type of sample, number of samples, instruments compared, outcomes/methodology used for the analysis and results.

\subsection{Quality Assessment}

The in vitro studies included were evaluated and classified as per the method design and risk of bias through the Grading of Recommendations Assessment, Development and Evaluation (GRADE) classification system [15].

The selected articles were graded for the evidence level as per the Oxford Center for Evidence-Based Medicine [16]. The studies were categorized into levels 1a, 1b, 1c, 2a, 2b, 2c, 3a, 3b, 4, and 5. The level of evidence 1 document Centre for Evidence-Based Medicine (CEBM) establishes some questions to systematize this process: therapy or prevention, etiology or harm, prognosis, diagnosis, differential diagnosis or study of the symptom's prevalence, and the economic and decision analysis.

The risk of bias of included clinical studies was assessed using the Newcastle Ottawa scale (NOS) [17].

Two reviewers (J.C., L.D.G.) independently evaluated the quality of studies based on the following parameters: Selection, Comparability and Outcome/Exposure. A maximum of 4 stars in selection domain, 2 stars in comparability domain and 4 stars in outcome/exposure domain were given. The included studies were qualified as "Good", "Fair" and "Poor" quality based on the total NOS score they achieved. Studies with a NOS score $\geq 7$ were considered good-quality studies.

Cochrane Handbook for Systematic Reviews of Interventions was employed for randomized clinical trials and randomized controlled trials [18].

The following quality criteria were assessed: sequence generation, allocation concealment, systematic differences in care provided to members of different study groups other than intervention under investigation (performance bias), systematic differences between groups in how outcomes were determined (detection bias), unequal loss of participants from study groups (attrition bias), within study selective outcome reporting (selective reporting bias), and other potential risks of bias.

A meta-analysis was not appropriate because of the methodological heterogeneity of the included studies. 


\section{Results}

\subsection{Bibliographic Search and Study Selection}

The initial database search yielded a total of 615 entries found in PubMed ${ }^{\circledR} /$ MEDLINE with 394 for "dental implant" AND "oral hygiene", 99 for "dental implant" AND curette, 18 for "dental implant" AND "ultrasonic scaler", 33 for "dental implant" AND "air polishing", 9 for "dental implant" AND "air flow", 40 for "dental implant" AND glycine, 10 for "dental implant" AND "sodium bicarbonate", 12 for "dental implant" AND erythritol.

A total of 319 entries were found in Cochrane Library with 129 for "dental implant" AND "oral hygiene", 2 for "dental implant" AND curette, 14 for "dental implant" AND "ultrasonic scaler", 38 for "dental implant" AND "air polishing", 116 for "dental implant" AND "air flow", 12 for "dental implant" AND glycine, 6 for "dental implant" AND "sodium bicarbonate", 2 for "dental implant" AND erythritol.

A flow chart that depicts the screening process is displayed in Figure 1.

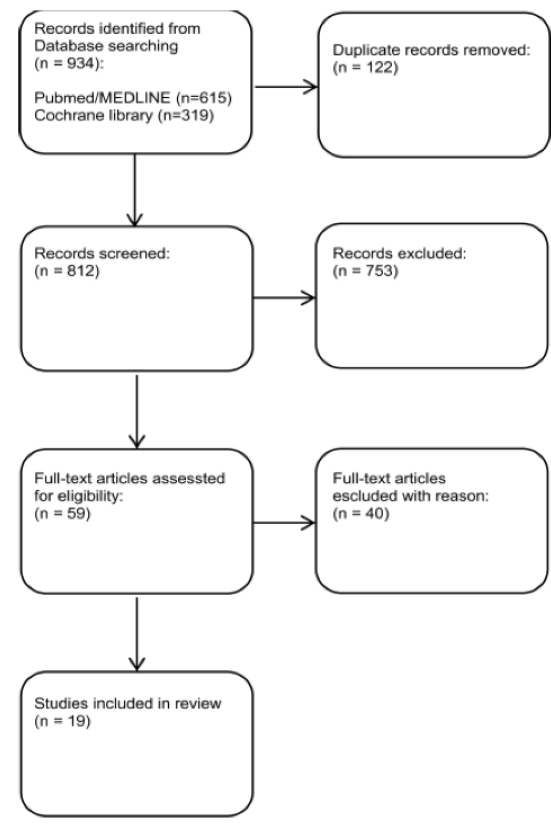

Figure 1. Preferred reporting of systematic reviews and meta-analyses (PRISMA) flow diagram related to bibliographic searching and study selection.

After excluding all duplicates, the total number of entries was reduced to 812 . A total of 753 articles were excluded after review of title and abstract. Hence, full text examination was conducted for 59 articles. A total of 40 additional articles were excluded after full text review and application of the eligibility criteria. The final selection consisted of 19 articles [6,7,11,13,19-33].

\subsection{Description of Included Studies}

Four of the included studies were conducted in Italy, two in USA, one in Canada, one in China, one in Taiwan, one in Australia, eight in Germany and one in Ireland. All the studies were in English language.

All the papers included explain the hygiene techniques used for the maintenance of implants, with studies on abutments or titanium disks, and compare which is the most effective in removing plaque and debris.

Detailed data for the 19 included studies are listed in Table 1. 
Table 1. Main characteristics of the included studies.

\begin{tabular}{|c|c|c|c|c|c|c|c|c|}
\hline Author(s) & $\begin{array}{c}\text { Publication } \\
\text { Year }\end{array}$ & Title & Study Design & Surface Treated & $\begin{array}{l}\text { Number of } \\
\text { Samples }\end{array}$ & $\begin{array}{l}\text { Instruments } \\
\text { Compared }\end{array}$ & Outcomes/Methodology & Results \\
\hline $\begin{array}{c}\text { Menini M, } \\
\text { Delucchi F, } \\
\text { Bagnasco F, Pera F, } \\
\text { Di Tullio N, } \\
\text { Pesce P. }\end{array}$ & 2021 & $\begin{array}{l}\text { Efficacy of } \\
\text { air-polishing } \\
\text { devices without } \\
\text { removal of } \\
\text { implant- } \\
\text { supported } \\
\text { full-arch } \\
\text { prostheses. }\end{array}$ & $\begin{array}{l}\text { Randomized } \\
\text { controlled trial }\end{array}$ & $\begin{array}{c}\text { Titanium implants } \\
\text { and conical } \\
\text { abutments }\end{array}$ & 357 & $\begin{array}{c}\text { Air polishing with } \\
\text { glycine powder, } \\
\text { ultrasonic device } \\
\text { with a } \\
\text { polyetheretherke- } \\
\text { tone fibre tip, } \\
\text { carbon fibre } \\
\text { curettes, sponge } \\
\text { floss }\end{array}$ & $\begin{array}{l}\text { Plaque Index, } \\
\text { peri-implant } \\
\text { spontaneous bleeding, } \\
\text { probing depth and } \\
\text { bleeding on probing }\end{array}$ & $\begin{array}{l}\text { Glycine powder } \\
\text { air polishing } \\
\text { resulted in a } \\
\text { significantly } \\
\text { higher reduction } \\
\text { in plaque around } \\
\text { implants. }\end{array}$ \\
\hline $\begin{array}{l}\text { Fletcher P, Linden } \\
\text { E, Cobb C, Zhao D, } \\
\text { Rubin J, Planzos P. }\end{array}$ & 2021 & $\begin{array}{c}\text { Efficacy of } \\
\text { Removal of } \\
\text { Residual Dental } \\
\text { Cement by Laser, } \\
\text { Ultrasonic Scalers, } \\
\text { and Titanium } \\
\text { Curette: An In } \\
\text { Vitro Study. }\end{array}$ & In vitro study & $\begin{array}{l}\text { Implants with } 3 \\
\text { different surface } \\
\text { textures }\end{array}$ & 39 & $\begin{array}{l}\text { Dental lasers, } \\
\text { ultrasonic scalers, } \\
\text { titanium curette }\end{array}$ & $\begin{array}{l}\text { Scanning electron } \\
\text { microscopy (SEM) }\end{array}$ & $\begin{array}{l}\text { No treatment } \\
\text { removed all } \\
\text { residual cement } \\
\text { from any of the } 3 \\
\text { implant surfaces. }\end{array}$ \\
\hline $\begin{array}{l}\text { Tong Z, Fu R, Zhu } \\
\text { W, Shi J, Yu M, } \\
\text { Si M. }\end{array}$ & 2021 & $\begin{array}{l}\text { Changes in the } \\
\text { surface } \\
\text { topography and } \\
\text { element } \\
\text { proportion of } \\
\text { clinically failed } \\
\text { SLA implants after } \\
\text { in vitro } \\
\text { debridement by } \\
\text { different methods. }\end{array}$ & In vitro study & Implants & 30 & $\begin{array}{l}\text { Physiologic saline } \\
\text { irrigation, glycine } \\
\text { powder, ethylene- } \\
\text { diaminetetraacetic } \\
\text { acid (EDTA), } \\
\text { ultrasonic scaler } \\
\text { with } \\
\text { polyetheretherke- } \\
\text { tone } \\
\text { (PEEK) tip }\end{array}$ & $\begin{array}{c}\text { Relative contaminated } \\
\text { area reduction (RCAR), } \\
\text { visual analogue scale } \\
\text { (VAS) and surface } \\
\text { roughness assessed } \\
\text { using } \\
\text { scanning electron } \\
\text { microscopy (SEM), } \\
\text { stereoscopic microscopy } \\
\text { (SM), white light } \\
\text { interferometry (WLI) }\end{array}$ & $\begin{array}{c}\text { PEEK tip } \\
\text { ultrasonic scaling } \\
\text { was more effective } \\
\text { in eliminating } \\
\text { visible } \\
\text { contamination. }\end{array}$ \\
\hline
\end{tabular}


Table 1. Cont.

\begin{tabular}{|c|c|c|c|c|c|c|c|c|}
\hline Author(s) & $\begin{array}{c}\text { Publication } \\
\text { Year }\end{array}$ & Title & Study Design & Surface Treated & $\begin{array}{l}\text { Number of } \\
\text { Samples }\end{array}$ & $\begin{array}{l}\text { Instruments } \\
\text { Compared }\end{array}$ & Outcomes/Methodology & Results \\
\hline $\begin{array}{c}\text { Di Tinco R, Bertani } \\
\text { G, Pisciotta A, } \\
\text { Bertoni L, } \\
\text { Bertacchini J, } \\
\text { Colombari B, } \\
\text { Conserva E, Blasi } \\
\text { E, Consolo U, } \\
\text { Carnevale G. }\end{array}$ & 2021 & $\begin{array}{l}\text { Evaluation of } \\
\text { Antimicrobial } \\
\text { Effect of } \\
\text { Air-Polishing } \\
\text { Treatments and } \\
\text { Their Influence on } \\
\text { Human Dental } \\
\text { Pulp Stem Cells } \\
\text { Seeded on } \\
\text { Titanium Disks. }\end{array}$ & In vitro study & Titanium disks & - & $\begin{array}{c}\text { Glycine and } \\
\text { tagatose powders }\end{array}$ & $\begin{array}{c}\text { Immunofluorescence } \\
\text { analyses }\end{array}$ & $\begin{array}{c}\text { Both the powders } \\
\text { have a great } \\
\text { in vitro cleaning } \\
\text { potential. }\end{array}$ \\
\hline $\begin{array}{c}\text { Matsubara VH, } \\
\text { Leong BW, Leong } \\
\text { MJL, Lawrence Z, } \\
\text { Becker T, } \\
\text { Quaranta A. }\end{array}$ & 2020 & $\begin{array}{l}\text { Cleaning potential } \\
\text { of different air } \\
\text { abrasive powders } \\
\text { and their impact } \\
\text { on implant surface } \\
\text { roughness. }\end{array}$ & In vitro study & Implants & 20 & $\begin{array}{c}\text { Sodium } \\
\text { bicarbonate, } \\
\text { glycine, Erythritol, } \\
\text { water alone }\end{array}$ & $\begin{array}{l}\text { Digital photography, } \\
\text { graphic software, } \\
\text { SEM and optical } \\
\text { profilometry }\end{array}$ & $\begin{array}{l}\text { Large-sized } \\
\text { powder showed } \\
\text { the greatest } \\
\text { cleaning capacity, } \\
\text { but caused more } \\
\text { alterations to the } \\
\text { implant surface. }\end{array}$ \\
\hline
\end{tabular}


Table 1. Cont.

\begin{tabular}{|c|c|c|c|c|c|c|c|c|}
\hline Author(s) & $\begin{array}{c}\text { Publication } \\
\text { Year }\end{array}$ & Title & Study Design & Surface Treated & $\begin{array}{c}\text { Number of } \\
\text { Samples }\end{array}$ & $\begin{array}{c}\text { Instruments } \\
\text { Compared }\end{array}$ & Outcomes/Methodology & Results \\
\hline $\begin{array}{c}\text { Menini M, Setti P, } \\
\text { Dellepiane E, } \\
\text { Zunino P, Pera P, } \\
\text { Pesce P }\end{array}$ & 2019 & $\begin{array}{l}\text { Comparison of biofilm } \\
\text { removal using glycine } \\
\text { air polishing versus } \\
\text { sodium bicarbonate } \\
\text { air polishing or hand } \\
\text { instrumentation on } \\
\text { full-arch fixed implant } \\
\text { rehabilitations: a } \\
\text { split-mouth study }\end{array}$ & $\begin{array}{l}\text { Randomized } \\
\text { controlled trial }\end{array}$ & $\begin{array}{l}\text { Titanium implants } \\
\text { and conical } \\
\text { abutments }\end{array}$ & 134 & $\begin{array}{l}\text { Glycine air } \\
\text { polishing, sodium } \\
\text { bicarbonate air } \\
\text { polishing, manual } \\
\text { scaling with } \\
\text { carbon-fiber } \\
\text { curette }\end{array}$ & $\begin{array}{l}\text { Spontaneous Bleeding } \\
\text { (SB), Plaque Index (PI) } \\
\text { were recorded before } \\
\text { and after hygiene. } \\
\text { Patient's satisfaction } \\
\text { towards the three } \\
\text { techniques was } \\
\text { analyzed by } \\
\text { questionnaires }\end{array}$ & $\begin{array}{c}\text { Sodium } \\
\text { bicarbonate air } \\
\text { polishing was the } \\
\text { most effective } \\
\text { method for plaque } \\
\text { reduction but was } \\
\text { more aggressive } \\
\text { on soft tissue and } \\
\text { was the least } \\
\text { preferred } \\
\text { treatment by } \\
\text { patients. Glycine } \\
\text { powder air } \\
\text { polishing was both } \\
\text { clinically effective } \\
\text { for plaque } \\
\text { removal and } \\
\text { highly accepted by } \\
\text { patients }\end{array}$ \\
\hline $\begin{array}{c}\text { Schmidt KE, } \\
\text { Auschill TM, } \\
\text { Sculean A, } \\
\text { Arweiler NB }\end{array}$ & 2019 & $\begin{array}{l}\text { Clinical evaluation of } \\
\text { non-surgical cleaning } \\
\text { modalities on } \\
\text { titanium dental } \\
\text { implants during } \\
\text { maintenance care: a } \\
\text { 1-year follow-up on } \\
\text { prosthodontic } \\
\text { superstructures }\end{array}$ & $\begin{array}{l}\text { Randomized } \\
\text { controlled trial }\end{array}$ & Implants & 32 & $\begin{array}{l}\text { Titanium curettes, } \\
\text { stainless steel } \\
\text { ultrasonic tip, } \\
\text { erythritol } \\
\text { air-polishing or } \\
\text { rubber cup } \\
\text { polishing }\end{array}$ & $\begin{array}{l}\text { Probing depths (PDs), } \\
\text { bleeding on probing } \\
\text { (BOP), modified } \\
\text { gingival (mucosal) } \\
\text { bleeding index (GBI) } \\
\text { around implants }\end{array}$ & $\begin{array}{c}\text { All tested } \\
\text { treatment } \\
\text { modalities yielded } \\
\text { comparable } \\
\text { clinical } \\
\text { improvements }\end{array}$ \\
\hline
\end{tabular}


Table 1. Cont.

\begin{tabular}{|c|c|c|c|c|c|c|c|c|}
\hline Author(s) & $\begin{array}{c}\text { Publication } \\
\text { Year }\end{array}$ & Title & Study Design & Surface Treated & $\begin{array}{l}\text { Number of } \\
\text { Samples }\end{array}$ & $\begin{array}{l}\text { Instruments } \\
\text { Compared }\end{array}$ & Outcomes/Methodology & Results \\
\hline $\begin{array}{l}\text { Schmidt KE, } \\
\text { Auschill TM, } \\
\text { Heumann C, } \\
\text { Frankenberger R, } \\
\text { Eick S, Sculean A, } \\
\text { Arweiler NB. }\end{array}$ & 2018 & $\begin{array}{c}\text { Clinical and } \\
\text { laboratory evaluation } \\
\text { of the effects of } \\
\text { different treatment } \\
\text { modalities on } \\
\text { titanium healing caps: } \\
\text { a randomized, } \\
\text { controlled clinical } \\
\text { trial. }\end{array}$ & $\begin{array}{l}\text { Randomized } \\
\text { controlled trial }\end{array}$ & $\begin{array}{c}\text { Titanium healing } \\
\text { caps }\end{array}$ & 72 & $\begin{array}{c}\text { Titanium curettes, } \\
\text { stainless steel } \\
\text { ultrasonic tip, } \\
\text { erythritol } \\
\text { air-polishing } \\
\text { powder, rubber } \\
\text { cup polishing }\end{array}$ & $\begin{array}{l}\text { Probing depths (PD), } \\
\text { bleeding on probing } \\
\text { (BOP), matrix } \\
\text { metalloproteinase } 8 \\
\text { (MMP-8), and } \\
\text { periopathogens }\end{array}$ & $\begin{array}{l}\text { All treatments } \\
\text { performed yielded } \\
\text { comparable } \\
\text { outcomes. }\end{array}$ \\
\hline $\begin{array}{c}\text { Lupi SM, Granati } \\
\text { M, Butera A, } \\
\text { Collesano V, } \\
\text { Rodriguez Y } \\
\text { Baena R. }\end{array}$ & 2017 & $\begin{array}{l}\text { Air-abrasive } \\
\text { debridement with } \\
\text { glycine powder } \\
\text { versus manual } \\
\text { debridement and } \\
\text { chlorhexidine } \\
\text { administration for the } \\
\text { maintenance of } \\
\text { peri-implant health } \\
\text { status: a six-month } \\
\text { randomized clinical } \\
\text { trial. }\end{array}$ & $\begin{array}{l}\text { Randomized } \\
\text { clinical trial }\end{array}$ & Implants & 88 & $\begin{array}{l}\text { Glycine powder, } \\
\text { manual } \\
\text { debridement and } \\
\text { clorexidine }\end{array}$ & $\begin{array}{c}\text { Plaque index (PI), } \\
\text { bleeding index (BOP), } \\
\text { probing depth (PD), } \\
\text { clinical attachment level } \\
\text { (CAL) and bleeding } \\
\text { score (BS) }\end{array}$ & $\begin{array}{l}\text { Treatment with } \\
\text { glycine seems } \\
\text { more effective } \\
\text { than the } \\
\text { traditional } \\
\text { treatment with } \\
\text { plastic curette and } \\
\text { chlorhexidine. }\end{array}$ \\
\hline $\begin{array}{l}\text { Al Ghazal L, } \\
\text { O'Sullivan J, } \\
\text { Claffey N, } \\
\text { Polyzois I. }\end{array}$ & 2017 & $\begin{array}{l}\text { Comparison of two } \\
\text { different techniques } \\
\text { used for the } \\
\text { maintenance of } \\
\text { peri-implant soft } \\
\text { tissue health: a pilot } \\
\text { randomized clinical } \\
\text { trial. }\end{array}$ & $\begin{array}{l}\text { Pilot randomized } \\
\text { clinical trial }\end{array}$ & Implants & 25 & $\begin{array}{l}\text { Low abrasive air } \\
\text { polishing powder } \\
\text { (Air-Flow®Perio, } \\
\text { EMS), titanium } \\
\text { curettes }\end{array}$ & $\begin{array}{l}\text { Bleeding on probing } \\
\text { (BOP), peri-implant } \\
\text { crevicular fluid analysis }\end{array}$ & $\begin{array}{l}\text { Both treatment } \\
\text { methods were } \\
\text { proven to be } \\
\text { effective in } \\
\text { reducing } \\
\text { peri-implant } \\
\text { inflammation and } \\
\text { preventing further } \\
\text { disease } \\
\text { progression. }\end{array}$ \\
\hline
\end{tabular}


Table 1. Cont.

\begin{tabular}{|c|c|c|c|c|c|c|c|c|}
\hline Author(s) & $\begin{array}{c}\text { Publication } \\
\text { Year }\end{array}$ & Title & Study Design & Surface Treated & $\begin{array}{l}\text { Number of } \\
\text { Samples }\end{array}$ & $\begin{array}{l}\text { Instruments } \\
\text { Compared }\end{array}$ & Outcomes/Methodology & Results \\
\hline $\begin{array}{l}\text { Al-Hashedi AA, } \\
\text { Laurenti M, } \\
\text { Benhamou V, } \\
\text { Tamimi F }\end{array}$ & 2017 & $\begin{array}{l}\text { Decontamination of } \\
\text { titanium implants } \\
\text { using physical } \\
\text { methods. }\end{array}$ & In vitro study & Implants & - & $\begin{array}{c}\text { Metal and plastic } \\
\text { curettes, Ti } \\
\text { brushes and Er: } \\
\text { YAG laser }\end{array}$ & $\begin{array}{c}\text { SEM, X-ray } \\
\text { photoelectron } \\
\text { spectroscopy, live-dead } \\
\text { assays }\end{array}$ & $\begin{array}{c}\text { Ti brushes were } \\
\text { more effective } \\
\text { than curettes } \\
\text { (metal or plastic) } \\
\text { and Er: YAG laser } \\
\text { in } \\
\text { decontaminating } \\
\text { Ti implant } \\
\text { surfaces. }\end{array}$ \\
\hline $\begin{array}{l}\text { Ziebolz D, Klipp S, } \\
\text { Schmalz G, } \\
\text { Schmickler J, } \\
\text { Rinke S, Kottmann } \\
\text { T, Fresmann S, } \\
\text { Einwag J }\end{array}$ & 2017 & $\begin{array}{l}\text { Comparison of } \\
\text { different maintenance } \\
\text { strategies within } \\
\text { supportive implant } \\
\text { therapy for prevention } \\
\text { of peri-implant } \\
\text { inflammation during } \\
\text { the first year after } \\
\text { implant restoration. A } \\
\text { randomized, dental } \\
\text { hygiene } \\
\text { practice-based } \\
\text { multicenter study }\end{array}$ & Clinical trial & Implants & 101 & $\begin{array}{c}\text { Manual curettes, a } \\
\text { sonic-driven scaler, } \\
\text { and a prophylaxis } \\
\text { brush, } \\
\text { chlorhexidine } \\
\text { (CHX) varnish, air } \\
\text { polishing with } \\
\text { glycine powder }\end{array}$ & $\begin{array}{l}\text { Peri-implant probing } \\
\text { depths (PPD), mucosal } \\
\text { recession (MR), and } \\
\text { bleeding on probing } \\
\text { (BOP) }\end{array}$ & $\begin{array}{l}\text { All strategies were } \\
\text { effective in } \\
\text { preventing } \\
\text { peri-implant } \\
\text { inflammation. } \\
\text { The supplemental } \\
\text { application of } \\
\text { chlorhexidine } \\
\text { varnish had no } \\
\text { significant } \\
\text { additional benefit }\end{array}$ \\
\hline $\begin{array}{l}\text { Chun KA, Kum } \\
\text { KY, Lee WC, Baek } \\
\text { SH, Choi HW, } \\
\text { Shon WJ. }\end{array}$ & 2017 & $\begin{array}{l}\text { Evaluation of the } \\
\text { safety and efficiency } \\
\text { of novel metallic } \\
\text { implant scaler tips } \\
\text { manufactured by the } \\
\text { powder injection } \\
\text { molding technique. }\end{array}$ & In vitro study & Titanium surfaces & & $\begin{array}{c}\text { Copper (CU), } \\
\text { bronze, } 316 \mathrm{~L} \\
\text { stainless steel (SS), } \\
\text { conventional } \\
\text { stainless steel } \\
\text { ultrasonic tips }\end{array}$ & $\begin{array}{l}\text { SEM, confocal laser } \\
\text { scanning microscopy } \\
\text { (CLSM) }\end{array}$ & $\begin{array}{l}\text { The efficiency of } \\
\text { the SS tip was } \\
\text { about } 3 \text { times } \\
\text { higher than that of } \\
\text { CU tip. }\end{array}$ \\
\hline
\end{tabular}


Table 1. Cont.

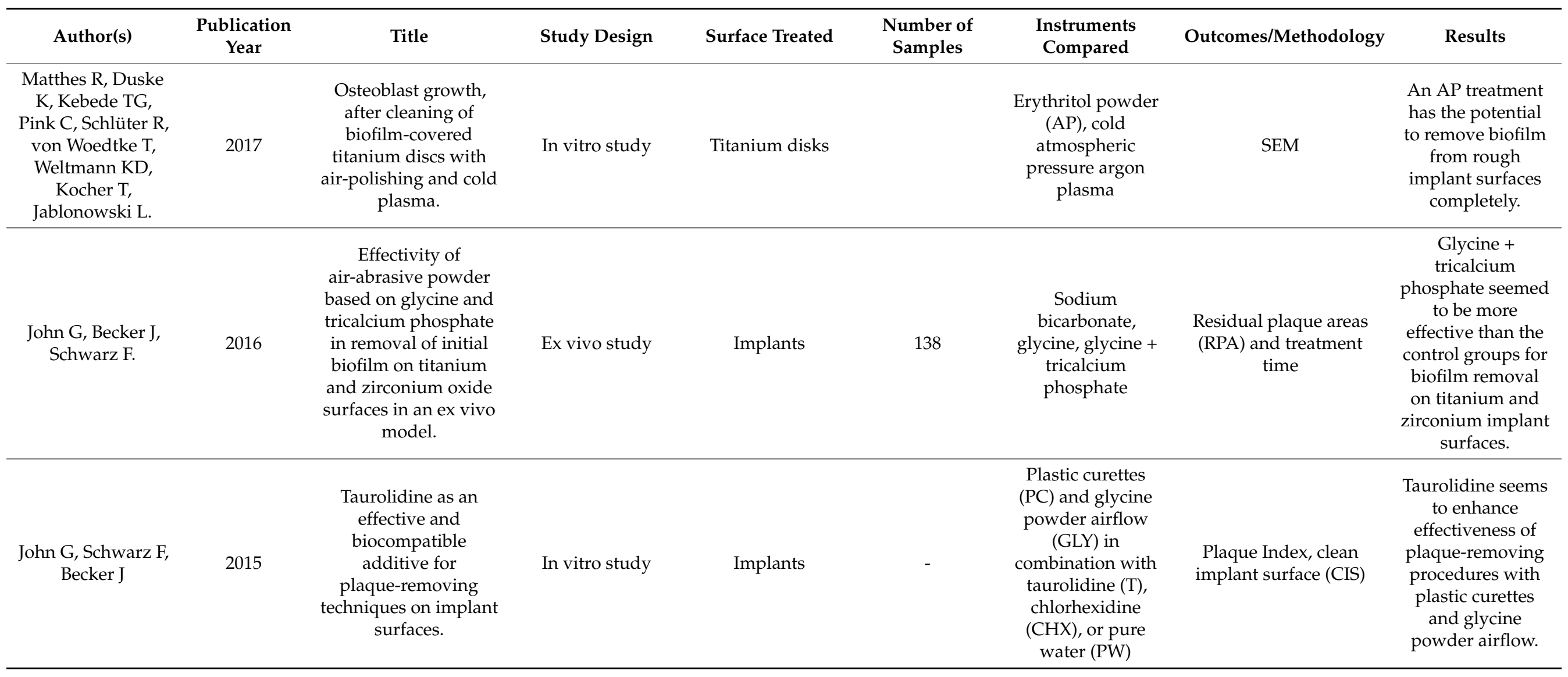


Table 1. Cont.

\begin{tabular}{|c|c|c|c|c|c|c|c|c|}
\hline Author(s) & $\begin{array}{c}\text { Publication } \\
\text { Year }\end{array}$ & Title & Study Design & Surface Treated & $\begin{array}{c}\text { Number of } \\
\text { Samples }\end{array}$ & $\begin{array}{c}\text { Instruments } \\
\text { Compared }\end{array}$ & Outcomes/Methodology & Results \\
\hline $\begin{array}{c}\text { Schmage P, Kahili } \\
\text { F, Nergiz I, } \\
\text { Scorziello TM, } \\
\text { Platzer U, } \\
\text { Pfeiffer P. }\end{array}$ & 2014 & $\begin{array}{c}\text { Cleaning effectiveness } \\
\text { of implant } \\
\text { prophylaxis } \\
\text { instruments. }\end{array}$ & In vitro study & Titanium disks & 80 & $\begin{array}{l}\text { Manual plastic } \\
\text { curette, manual } \\
\text { carbon } \\
\text { fiber-reinforced } \\
\text { plastic (CFRP) } \\
\text { curette, } \\
\text { sonic-driven } \\
\text { prophylaxis brush, } \\
\text { rotating rubber } \\
\text { cup with } \\
\text { prophylaxis paste, } \\
\text { sonic-driven } \\
\text { polyether ether } \\
\text { ketone (PEEK) } \\
\text { plastic tip, } \\
\text { ultrasonic-driven } \\
\text { PEEK plastic tip, } \\
\text { and air polishing } \\
\text { with amino acid } \\
\text { (glycine) powder }\end{array}$ & Light microscopy & $\begin{array}{l}\text { The cleaning } \\
\text { effectiveness of } \\
\text { the plastic curette } \\
\text { was significantly } \\
\text { lower.Superior } \\
\text { results, with less } \\
\text { than } 4 \% \text { of the } \\
\text { biofilm remaining, } \\
\text { were obtained for } \\
\text { both oscillating } \\
\text { PEEK plastic tips } \\
\text { and air polishing. }\end{array}$ \\
\hline $\begin{array}{c}\text { Swierkot K, } \\
\text { Brusius M, } \\
\text { Leismann D, } \\
\text { Nonnenmacher C, } \\
\text { Nüsing R, } \\
\text { Lubbe D, } \\
\text { Schade-Brittinger } \\
\text { C, Mengel R }\end{array}$ & 2013 & $\begin{array}{l}\text { Manual versus } \\
\text { sonic-powered } \\
\text { toothbrushing for } \\
\text { plaque reduction in } \\
\text { patients with dental } \\
\text { implants: an } \\
\text { explanatory } \\
\text { randomised } \\
\text { controlled trial. }\end{array}$ & $\begin{array}{l}\text { Randomized } \\
\text { controlled trial }\end{array}$ & Implants & - & $\begin{array}{l}\text { Sonic toothbrush, } \\
\text { manual } \\
\text { toothbrush }\end{array}$ & $\begin{array}{c}\text { Real-time polymerase } \\
\text { chain reaction, } \\
\text { chromatography- } \\
\text { electrospray } \\
\text { spectrometry }\end{array}$ & $\begin{array}{l}\text { The plaque index } \\
\text { difference between } \\
\text { baseline and } 12 \\
\text { months at } \\
\text { implants showed } \\
\text { no significant } \\
\text { difference between } \\
\text { sonic or manual } \\
\text { toothbrushing. }\end{array}$ \\
\hline
\end{tabular}




\subsection{Excluded Studies}

Out of 59 papers for which the full text was analyzed, 40 articles were excluded from the systematic review (Appendix A, Table A1) [4,5,10,12,34-69], the main reason for exclusion being not focusing on efficacy of removing plaque and debris, not comparing different hygiene instruments and reproducing peri-implant bone defects.

\subsection{Quality Assessment of Included Studies}

The risk of bias of included clinical studies was assessed using the Newcastle Ottawa scale (NOS). Outcomes are reported in Table 2.

Table 2. Risk of bias for clinical studies included in the present systematic review according to the NOS-Newcastle-Ottawa Scale.

\begin{tabular}{ccccc}
\hline Study & Selection & Comparability & Outcome/Exposure & NOS Score \\
\hline Ziebolz et al. 2017 & $\bullet \bullet \circ \circ$ & $\bullet \circ$ & $\circ \bullet \bullet$ & 5 \\
John et al. 2016 & $\bullet \bullet \circ$ & $\bullet \circ$ & $\circ \bullet \bullet$ & 5 \\
\hline
\end{tabular}

The risk of bias for the randomized clinical and controlled trials included in the present systematic review was assessed using the Cochrane Handbook for Systematic Reviews of Interventions. Outcomes are reported in Table 3.

Table 3. Risk of bias for the randomized clinical and controlled trials included in the present systematic review according to Cochrane Handbook for Systematic Reviews of Interventions.

\begin{tabular}{cccccccc}
\hline Study & $\begin{array}{c}\text { Selection } \\
\text { Bias } \\
\text { Sequence } \\
\text { Generation }\end{array}$ & $\begin{array}{c}\text { Selection } \\
\text { Bias } \\
\text { Allocation } \\
\text { Concealment }\end{array}$ & $\begin{array}{c}\text { Performance } \\
\text { Bias }\end{array}$ & $\begin{array}{c}\text { Detection } \\
\text { Bias }\end{array}$ & $\begin{array}{c}\text { Attrition } \\
\text { Bias }\end{array}$ & $\begin{array}{c}\text { Selective } \\
\text { Reporting } \\
\text { Bias }\end{array}$ & $\begin{array}{c}\text { Other } \\
\text { Potential } \\
\text { Risk } \\
\text { of Bias }\end{array}$ \\
\hline Menini et al., 2021 & Low & Low & High & Unclear & Low & Low & Low \\
\hline Menini et al., 2019 & Low & Low & High & Low & Low & Low & Low \\
\hline Schmidt et al., 2019 & Low & High & High & Low & High & Low & Low \\
\hline Schmidt et al., 2018 & Low & High & High & Low & High & Low & Low \\
\hline Lupi et al., 2017 & Low & Low & High & Unclear & Low & Low & Low \\
\hline Al Ghazal et al., 2017 & Low & High & High & Unclear & Low & Low & Low \\
\hline Swierkot et al., 2013 & Low & Unclear & High & Unclear & Low & Low & Low \\
\hline
\end{tabular}

Evaluation and classification of included in vitro studies was assessed using the Grading of Recommendations Assessment, Development and Evaluation (GRADE), graduation for the evidence level was assessed using the Oxford Center for Evidence-Based Medicine. Outcomes are reported in Table 4. 
Table 4. Evaluation and classification of included in vitro studies through the Grading of Recommendations Assessment, Development and Evaluation (GRADE), graduation for the evidence level as per the Oxford Center for Evidence-Based Medicine.

\begin{tabular}{|c|c|c|c|}
\hline \multirow[b]{2}{*}{ Study } & \multirow[b]{2}{*}{ Reference Number } & \multicolumn{2}{|c|}{ Level of Evidence Oxford } \\
\hline & & $\begin{array}{c}\text { Degree of } \\
\text { Recommendation }\end{array}$ & Level of Evidence \\
\hline Fletcher et al., 2021 & 19 & B & $2 \mathrm{C}$ \\
\hline Tong et al., 2021 & 20 & B & $2 \mathrm{C}$ \\
\hline Di Tinco et al., 2021 & 21 & B & $2 \mathrm{C}$ \\
\hline Matsubara et al., 2020 & 13 & B & $2 \mathrm{C}$ \\
\hline Sirinirund et al., 2019 & 22 & B & $2 \mathrm{C}$ \\
\hline Al-Hashedi et al., 2017 & 26 & B & $2 \mathrm{C}$ \\
\hline Chun et al., 2017 & 28 & B & $2 \mathrm{C}$ \\
\hline Matthes et al., 2017 & 29 & B & $2 \mathrm{C}$ \\
\hline John et al., 2015 & 31 & B & $2 \mathrm{C}$ \\
\hline Schmage et al., 2014 & 32 & B & $2 \mathrm{C}$ \\
\hline
\end{tabular}

\section{Discussion}

Curette, scalers and air polishing are common and effective strategies applied for professional oral hygiene in order to prevent peri-implant inflammation [27].

However, as supported by the studies included in the present review, instrumentation of implant and prosthodontic components with curettes for biofilm management might present some disadvantages and air polishing devices with low-abrasive powders are increasingly gaining acceptance. Fifteen out of 19 studies included investigated the efficacy of air-polishing devices showing favorable outcomes. In particular, professional oral hygiene using glycine powder air polishing has been demonstrated to be clinically effective for plaque removal on dental implants but also highly accepted by patients [6,7]. It may be considered a viable method to remove plaque from dental implants because glycine is less aggressive than powders like sodium bicarbonate powder, that might cause more alterations to the implant surface and is more aggressive on soft tissue, depending on its larger granulometry $[7,13]$.

Glycine powder air polishing resulted in a significantly higher reduction in plaque around implants [7,11], and effective in reducing peri-implant inflammation [25]. In vitro studies showed that also tagatose powders could have cleaning potential with great results [21]. In addition, the combination of glycine and taurolidine could enhance effectiveness of plaque-removing procedures [31]. A study by John et al. combined glycine with tricalcium phosphate: they seemed to be more effective than the control groups for biofilm removal on titanium and zirconium implant surfaces [30].

The application of air polishing with erythritol powder is another more recent alternative. It has the potential to efficiently remove biofilm from rough implant surfaces [29] and yielded clinical outcomes comparable with titanium curettes, stainless steel ultrasonic tip, or rubber cup polishing [23,24].

Moving to other oral hygiene instruments, Fletcher et al. demonstrated that dental lasers, ultrasonic scalers and titanium curette didn't remove all residual cement from any of the 3 implant surfaces in their in vitro study [19]. PEEK tip or stainless steel (SS) tip ultrasonic scaling are more effective in eliminating visible contamination according to other authors $[20,22,28]$.

Further studies are needed about the application of dental laser such as Er: YAG laser in decontaminating Ti implant surfaces [26]. 
In order to help to lead to better biofilm removal and to reinforce proper oral and correct self-care regimens, Swierkot et al. compared plaque index after 12 months of sonic vs. manual toothbrushing: the results showed no significant difference. [33]

In choosing the ideal instrument, the dentist and the dental hygienist must also consider other aspects in addition to effectiveness in plaque deposits removal, such as the possible harmful production of roughness of the implant surface or of the prosthetic surfaces, or the release of debris on the implant prosthodontic surface.

This review was not specifically realized to investigate this topic, however, in the study by Schmage et al. no traces have been determined on the treated surfaces after manipulation with acrylic curettes even if the cleaning effectiveness of the plastic curette was significantly lower than machine-driven instruments [32].

About this last topic, a final consideration should be done about excluded studies. In particular, regarding the concept of cavitation. Vyas et al. [42,43] demonstrated the effectiveness of cavitation bubbles in biofilms removal. More studies are necessary to validate this technique that sounds very interesting for the capability to prevent mechanical damages of implant and prosthetic surfaces. However, this theme is debated.

Schmidt et al. [52] supported the idea that one-time instrumentation $(30 \mathrm{sec})$ is not able to damage implant surface independently by the instruments used, excluding steel curettes that created damages.

While Schmage et al. [69] and Park et al. [68] sustained that alterations of the implant surfaces were strongly dependent on the implant cleaning method used.

\section{Conclusions}

Routine assessment of peri-implant tissue changes and mechanical biofilm removal with the use of appropriate armamentarium is essential in order to avoid damage to implant-prosthodontic components and maintain peri-implant tissues over time. Air polishing systems allow effective, optimal plaque and biofilm management, and might be viable alternatives to traditional hand instruments (curettes, scalers) and classic rotating instruments used for polishing. Further studies on new and different hygiene devices will improve the knowledge on the topic in order to develop standardized protocols and understand the specific indications for different types of implant-supported rehabilitations. This will help the clinicians in the choice of the best professional oral hygiene instruments and techniques to be applied in each specific clinical situation.

Author Contributions: Conceptualization: J.C., L.D.G. and D.B.; methodology, J.C., L.D.G. and D.B.; software, J.C. and L.D.G.; validation, J.C., L.D.G., M.M., F.M. and D.B.; formal analysis, J.C., L.D.G. and D.B.; investigation, J.C. and L.D.G.; resources, J.C. and L.D.G.; data curation, J.C. and L.D.G.; writing-original draft preparation, J.C. and L.D.G.; writing—review and editing, J.C. and L.D.G.; visualization, J.C., L.D.G., M.M., F.M. and D.B.; supervision, J.C., L.D.G., M.M., F.M. and D.B.; project administration, J.C., L.D.G., M.M., F.M. and D.B. All authors have read and agreed to the published version of the manuscript.

Funding: This research received no external funding.

Institutional Review Board Statement: Not applicable.

Informed Consent Statement: Not applicable.

Data Availability Statement: Data supporting reported results can be found on PubMed ${ }^{\circledR} /$ MEDLINE.

Conflicts of Interest: The authors declare no conflict of interest. 


\section{Appendix A}

Table A1. Table reporting the 40 excluded studies and reasons for exclusion.

\begin{tabular}{|c|c|}
\hline Study & Reason for Exclusion \\
\hline Amate-Fernández et al., 2021 & Not focused on efficacy \\
\hline Salles et al., 2021 & Home oral hygiene \\
\hline Iatrou et al., 2021 & Simulation of peri-implant defects \\
\hline Salles et al., 2021 & Home oral hygiene \\
\hline Hu et al., 2020 & Not focused on efficacy \\
\hline Mensi et al.2020 & Simulation of peri-implant defects \\
\hline Hu et al., 2020 & Not focused on efficacy \\
\hline Gümüş et al., 2020 & Not focused on efficacy \\
\hline Vyas et al., 2020 & No instrument comparison \\
\hline Vyas et al., 2020 & No instrument comparison \\
\hline Huang et al., 2019 & Not focused on efficacy \\
\hline Cha et al., 2019 & Not focused on efficacy \\
\hline Keim et al., 2019 & Simulation of peri-implant defects \\
\hline Biazussi et al., 2019 & Not focused on efficacy \\
\hline Harrel et al., 2019 & Not focused on efficacy \\
\hline Takagi et al., 2018 & Not focused on efficacy \\
\hline Cao et al., 2018 & Not focused on efficacy \\
\hline Larsen et al., 2017 & Not focused on efficacy \\
\hline Quintero et al., 2017 & Not focused on efficacy \\
\hline Schmidt et al., 2017 & Not focused on efficacy \\
\hline Hakki et al., 2017 & Not focused on efficacy \\
\hline Kister et al., 2017 & Not focused on efficacy \\
\hline Ronay et al., 2017 & Simulation of peri-implant defects \\
\hline Bertoldi et al., 2017 & Not focused on efficacy \\
\hline Tastepe et al., 2017 & No instrument comparison \\
\hline Chen et al., 2016 & Not focused on efficacy \\
\hline Rios et al., 2016 & Not focused on efficacy \\
\hline Lang et al., 2016 & Not focused on efficacy \\
\hline Park et al., 2015 & Not focused on efficacy \\
\hline Yang et al., 2015 & Not focused on efficacy \\
\hline Anastassiadis et al., 2015 & Not focused on efficacy \\
\hline Sahrmann et al., 2015 & Simulation of peri-implant defects \\
\hline Menini et al., 2015 & Not focused on efficacy \\
\hline Schmage et al., 2012 & Not focused on efficacy \\
\hline Park et al., 2013 & Not focused on efficacy \\
\hline
\end{tabular}


Table A1. Cont.

\begin{tabular}{cc}
\hline Study & Reason for Exclusion \\
\hline Sahrmann et al., 2013 & Simulation of peri-implant defects \\
\hline Mussano et al., 2013 & Not focused on efficacy \\
\hline Nemer Vieira et al., 2012 & Not focused on efficacy \\
\hline Park et al., 2012 & Not focused on efficacy \\
\hline Mann et al., 2012 & Not focused on efficacy \\
\hline
\end{tabular}

\section{References}

1. Baldi, D.; Menini, M.; Colombo, J.; Lertora, E.; Pera, P. Evaluation of a New Ultrasonic Insert for Prosthodontic Preparation. Int. J. Prosthodont. 2017, 30, 496-498. [CrossRef] [PubMed]

2. Baldi, D.; Colombo, J.; Robiony, M.; Menini, M.; Bisagni, E.; Pera, P. Temperature variations in pulp chamber: An in-vitro comparison between ultrasonic and rotating instruments in tooth preparation. Part 1. Minerva Stomatol. 2020, 69, 14-20. [CrossRef] [PubMed]

3. Baldi, D.; Colombo, J.; Stacchi, C.; Menini, M.; Oronos, A.; Pera, P. Pulp vitality during ultrasonic tooth preparation. Part 2. Minerva Stomatol. 2020, 69, 21-26. [CrossRef] [PubMed]

4. Mann, M.; Parmar, D.; Walmsley, A.D.; Lea, S.C. Effect of plastic covered ultrasonic scalers on titanium implant surfaces. Clin. Oral Implant. Res. 2012, 23, 76-82. [CrossRef] [PubMed]

5. Menini, M.; Piccardo, P.; Baldi, D.; Dellepiane, E.; Pera, P. Morphological and chemical characteristics of different titanium surfaces treated by bicarbonate and glycine powder air abrasive systems. Implant. Dent. 2015, 24, 47-56. [CrossRef]

6. Menini, M.; Setti, P.; Dellepiane, E.; Zunino, P.; Pera, P.; Pesce, P. Comparison of biofilm removal using glycine air polishing versus sodium bicarbonate air polishing or hand instrumentation on full-arch fixed implant rehabilitations: A split-mouth study. Quintessence Int. 2019, 8, 2-10.

7. Menini, M.; Delucchi, F.; Bagnasco, F.; Pera, F.; Di Tullio, N.; Pesce, P. Efficacy of air-polishing devices without removal of implant-supported full-arch prostheses. Int. J. Oral Implantol. 2021, 14, 401-416.

8. Sison, S.G. Implant Maintenance and the Dental Hygienist. Available online: http://www.kiezenoftrekken.nl/downloads/ supimplant.pdf (accessed on 30 April 2021).

9. Fody, A.; Marsh, L. Importance of Implant Maintenance. Dimens. Dent. Hyg. 2020, 18, 16-18, 21. Available online: https: // dimensionsofdentalhygiene.com/article/importance-of-implant-maintenance/ (accessed on 30 April 2021).

10. Lang, M.S.; Cerutis, D.R.; Miyamoto, T.; Nunn, M.E. Cell Attachment Following Instrumentation with Titanium and Plastic Instruments, Diode Laser, and Titanium Brush on Titanium, Titanium-Zirconium, and Zirconia Surfaces. Int. J. Oral Maxillofac. Implant. 2016, 31, 799-806. [CrossRef]

11. Lupi, S.M.; Granati, M.; Butera, A.; Collesano, V.; Baena, R.R.Y. Air-abrasive debridement with glycine powder versus manual debridement and chlorhexidine administration for the maintenance of peri-implant health status: A six-month randomized clinical trial. Int. J. Dent. Hyg. 2016, 15, 287-294. [CrossRef]

12. Yang, S.-M.; Park, J.-B.; Ko, Y. Use of confocal microscopy for quantification of plastic remnants on rough titanium after instrumentation and evaluation of efficacy of removal. Int. J. Oral Maxillofac. Implant. 2015, 30, 519-525. [CrossRef]

13. Matsubara, V.H.; Leong, B.W.; Leong, M.J.L.; Lawrence, Z.; Becker, T.; Quaranta, A. Cleaning potential of different air abrasive powders and their impact on implant surface roughness. Clin. Implant. Dent. Relat. Res. 2020, 22, 96-104. [CrossRef] [PubMed]

14. Moher, D.; Liberati, A.; Tetzlaff, J.; Altman, D.G.; PRISMA Group. Preferred reporting items for systematic reviews and meta-analyses: The PRISMA statement. PLoS Med. 2009, 6, e1000097. [CrossRef]

15. Guyatt, G.H.; Oxman, A.D.; Vist, G.E.; Kunz, R.; Falck-Ytter, Y.; Alonso-Coello, P. GRADE: An emerging consensus on rating quality of evidence and strength of recommendations. BMJ 2008, 336, 924-926. [CrossRef]

16. Howick, J.; Chalmers, I.; Glasziou, P.; Greenhalgh, T.; Heneghan, C.; Liberati, A. The 2011 Oxford CEBM Evidence Levels of Evidence (Introductory Document); Oxford Center for Evidence-Based Medicine: Oxford, UK, 2011; pp. 1-3.

17. Wells, G.A.; Shea, B.; O'Connell, D.; Peterson, J.; Welch, V.; Losos, M.; Tugwell, P. The Newcastle-Ottawa Scale (NOS) for Assessing the Quality of Non-Randomized Studies in Meta-Analysis; Ottawa Hospital Research Institute: Ottawa, ON, Canada, 2000; Available online: http:/ / www.ohri.ca/programs/clinical_epidemiology / oxford.asp (accessed on 30 September 2021).

18. Higgins, J.P.T.; Green, S. (Eds.) Cochrane Handbook for Systematic Reviews of Interventions Version 5.1.0 [updated 11]. In The Cochrane Collaboration; 20 March 2011; Available online: www.handbook.cochrane.org (accessed on 30 September 2021).

19. Fletcher, P.; Linden, E.; Cobb, C.; Zhao, D.; Rubin, J.; Planzos, P. Efficacy of Removal of Residual Dental Cement by Laser, Ultrasonic Scalers, and Titanium Curette: An In Vitro Study. Compend Contin Educ Dent. 2021, 42, e5-e9.

20. Tong, Z.; Fu, R.; Zhu, W.; Shi, J.; Yu, M.; Si, M. Changes in the surface topography and element proportion of clinically failed SLA implants after in vitro debridement by different methods. Clin. Oral Implant. Res. 2021, 32, 263-273. [CrossRef] 
21. Di Tinco, R.; Bertani, G.; Pisciotta, A.; Bertoni, L.; Bertacchini, J.; Colombari, B.; Conserva, E.; Blasi, E.; Consolo, U.; Carnevale, G. Evaluation of Antimicrobial Effect of Air-Polishing Treatments and Their Influence on Human Dental Pulp Stem Cells Seeded on Titanium Disks. Int. J. Mol. Sci. 2021, 22, 865. [CrossRef] [PubMed]

22. Sirinirund, B.; Garaicoa-Pazmino, C.; Wang, H.L. Effects of Mechanical Instrumentation with Commercially Available Instruments Used in Supportive Peri-implant Therapy: An In Vitro Study. Int. J. Oral Maxillofac. Implant. 2019, 34, 1370-1378. [CrossRef]

23. Schmidt, K.E.; Auschill, T.M.; Sculean, A.; Arweiler, N.B. Clinical evaluation of non-surgical cleaning modalities on titanium dental implants during maintenance care: A 1-year follow-up on prosthodontic superstructures. Clin. Oral Investig. 2019, 23, 1921-1930. [CrossRef] [PubMed]

24. Schmidt, K.E.; Auschill, T.M.; Heumann, C.; Frankenberger, R.; Eick, S.; Sculean, A.; Arweiler, N.B. Clinical and laboratory evaluation of the effects of different treatment modalities on titanium healing caps: A randomized, controlled clinical trial. Clin. Oral Investig. 2018, 22, 2149-2160. [CrossRef]

25. Al Ghazal, L.; O'Sullivan, J.; Claffey, N.; Polyzois, I. Comparison of two different techniques used for the maintenance of peri-implant soft tissue health: A pilot randomized clinical trial. Acta Odontol. Scand. 2017, 75, 542-549. [CrossRef]

26. Al-Hashedi, A.A.; Laurenti, M.; Benhamou, V.; Tamimi, F. Decontamination of titanium implants using physical methods. Clin. Oral Implant. Res. 2017, 28, 1013-1021. [CrossRef] [PubMed]

27. Ziebolz, D.; Klipp, S.; Schmalz, G.; Schmickler, J.; Rinke, S.; Kottmann, T.; Fresmann, S.; Einwag, J. Comparison of different maintenance strategies within supportive implant therapy for prevention of peri-implant inflammation during the first year after implant restoration. A randomized, dental hygiene practice-based multicenter study. Am. J. Dent. 2017, 30, $190-196$.

28. Chun, K.A.; Kum, K.Y.; Lee, W.C.; Baek, S.H.; Choi, H.W.; Shon, W.J. Evaluation of the safety and efficiency of novel metallic implant scaler tips manufactured by the powder injection molding technique. BMC Oral Health 2017, 17, 110. [CrossRef]

29. Matthes, R.; Duske, K.; Kebede, T.G.; Pink, C.; Schlüter, R.; von Woedtke, T.; Weltmann, K.D.; Kocher, T.; Jablonowski, L. Osteoblast growth, after cleaning of biofilm-covered titanium discs with air-polishing and cold plasma. J. Clin. Periodontol. 2017, 44, 672-680. [CrossRef] [PubMed]

30. John, G.; Becker, J.; Schwarz, F. Effectivity of air-abrasive powder based on glycine and tricalcium phosphate in removal of initial biofilm on titanium and zirconium oxide surfaces in an ex vivo model. Clin. Oral Investig. 2016, 20, 711-719. [CrossRef]

31. John, G.; Schwarz, F.; Becker, J. Taurolidine as an effective and biocompatible additive for plaque-removing techniques on implant surfaces. Clin. Oral Investig. 2015, 19, 1069-1077. [CrossRef] [PubMed]

32. Schmage, P.; Kahili, F.; Nergiz, I.; Scorziello, T.M.; Platzer, U.; Pfeiffer, P. Cleaning effectiveness of implant prophylaxis instruments. Int. J. Oral Maxillofac. Implant. 2014, 29, 331-337. [CrossRef]

33. Swierkot, K.; Brusius, M.; Leismann, D.; Nonnenmacher, C.; Nüsing, R.; Lubbe, D.; Schade-Brittinger, C.; Mengel, R. Manual versus sonic-powered toothbrushing for plaque reduction in patients with dental implants: An explanatory randomised controlled trial. Eur. J. Oral Implantol. 2013, 6, 133-144.

34. Amate-Fernández, P.; Figueiredo, R.; Blanc, V.; Àlvarez, G.; León, R.; Valmaseda-Castellón, E. Erythritol-enriched powder and oral biofilm regrowth on dental implants: An in vitro study. Med. Oral Patol. Oral Cir. Bucal. 2021, 26, e602-e610. [CrossRef]

35. Salles, M.M.; de Cássia Oliveira, V.; Macedo, A.P.; Silva-Lovato, C.H.; de Freitas de Oliveira Paranhos, H. Effectiveness of Brushing Associated with Oral Irrigation in Maintenance of Peri-Implant Tissues and Overdentures: Clinical Parameters and Patient Satisfaction. J. Oral Implantol. 2021, 47, 117-123. [CrossRef]

36. Iatrou, P.; Chamilos, C.; Nickles, K.; Ratka, C.; Eickholz, P.; Petsos, H. In Vitro Efficacy of Three Different Nonsurgical Implant Surface Decontamination Methods in Three Different Defect Configurations. Int. J. Oral Maxillofac. Implant. 2021, 36, 271-280. [CrossRef] [PubMed]

37. Salles, M.M.; Oliveira, V.C.; Macedo, A.P.; do Nascimento, C.; Silva-Lovato, C.H.; Paranhos, H.F.O. Brushing associated with oral irrigation in maintaining implants and overdentures hygiene-A randomized clinical trial. Odontology 2021, 109, 284-294. [CrossRef] [PubMed]

38. Hu, J.; Atsuta, I.; Ayukawa, Y.; Zhou, T.; Narimatsu, I.; Koyano, K. Effect of titanium or zirconia implant abutments on epithelial attachments after ultrasonic cleaning. J. Oral Sci. 2020, 62, 331-334. [CrossRef] [PubMed]

39. Mensi, M.; Viviani, L.; Agosti, R.; Scotti, E.; Garzetti, G.; Calza, S. Comparison between four different implant surface debridement methods: An in-vitro experimental study. Minerva Stomatol. 2020, 69, 286-294. [CrossRef]

40. Hu, J.; Atsuta, I.; Ayukawa, Y.; Zhou, X.; Dwi Rakhmatia, Y.; Koyano, K. The impact of surface alteration on epithelial tissue attachment after the mechanical cleaning of titanium or zirconia surface. J. Oral Rehabil. 2020, 47, 1065-1076. [CrossRef]

41. Gümüş, K.Ç.; Ustaoğlu, G.; Kara, L.; Ercan, E.; Albayrak, Ö.; Tunali, M. Nano-Hydroxyapatite Airborne-Particle Abrasion System as an Alternative Surface Treatment Method on Intraorally Contaminated Titanium Discs. Int. J. Periodontics Restorative Dent. 2020, 40, e179-e187. [CrossRef]

42. Vyas, N.; Sammons, R.L.; Kuehne, S.A.; Johansson, C.; Stenport, V.; Wang, Q.X.; Walmsley, A.D. The effect of standoff distance and surface roughness on biofilm disruption using cavitation. PLoS ONE 2020, 15, e0236428. [CrossRef]

43. Vyas, N.; Grewal, M.; Kuehne, S.A.; Sammons, R.L.; Walmsley, A.D. High speed imaging of biofilm removal from a dental implant model using ultrasonic cavitation. Dent. Mater. 2020, 36, 733-743. [CrossRef] [PubMed]

44. Huang, Y.S.; Hung, C.Y.; Huang, H.H. Surface changes and bacterial adhesion on implant abutment materials after various clinical cleaning procedures. J. Chin. Med. Assoc. 2019, 82, 643-650. [CrossRef] 
45. Cha, J.K.; Paeng, K.; Jung, U.W.; Choi, S.H.; Sanz, M.; Sanz-Martín, I. The effect of five mechanical instrumentation protocols on implant surface topography and roughness: A scanning electron microscope and confocal laser scanning microscope analysis. Clin. Oral Implant. Res. 2019, 30, 578-587. [CrossRef]

46. Keim, D.; Nickles, K.; Dannewitz, B.; Ratka, C.; Eickholz, P.; Petsos, H. In vitro efficacy of three different implant surface decontamination methods in three different defect configurations. Clin. Oral Implant. Res. 2019, 30, 550-558. [CrossRef] [PubMed]

47. Biazussi, B.R.; Perrotti, V.; D'Arcangelo, C.; Elias, C.N.; Bianchini, M.A.; Tumedei, M.; de Vasconcellos, D.K. Evaluation of the Effect of Air Polishing With Different Abrasive Powders on the Roughness of Implant Abutment Surface: An In Vitro Study. J. Oral Implantol. 2019, 45, 202-206. [CrossRef] [PubMed]

48. Harrel, S.K.; Wilson, T.G., Jr.; Pandya, M.; Diekwisch, T.G.H. Titanium particles generated during ultrasonic scaling of implants. J. Periodontol. 2019, 90, 241-246. [CrossRef]

49. Takagi, T.; Aoki, A.; Ichinose, S.; Taniguchi, Y.; Tachikawa, N.; Shinoki, T.; Meinzer, W.; Sculean, A.; Izumi, Y. Effective removal of calcified deposits on microstructured titanium fixture surfaces of dental implants with erbium lasers. J. Periodontol. 2018, 89, 680-690. [CrossRef]

50. Cao, J.; Wang, T.; Pu, Y.; Tang, Z.; Meng, H. Influence on proliferation and adhesion of human gingival fibroblasts from different titanium surface decontamination treatments: An in vitro study. Arch. Oral Biol. 2018, 87, 204-210. [CrossRef]

51. Quintero, D.G.; Taylor, R.B.; Miller, M.B.; Merchant, K.R.; Pasieta, S.A. Air-Abrasive Disinfection of Implant Surfaces in a Simulated Model of Periimplantitis. Implant. Dent. 2017, 26, 423-428. [CrossRef]

52. Schmidt, K.E.; Auschill, T.M.; Heumann, C.; Frankenberger, R.; Eick, S.; Sculean, A.; Arweiler, N.B. Influence of different instrumentation modalities on the surface characteristics and biofilm formation on dental implant neck, in vitro. Clin. Oral Implant. Res. 2017, 28, 483-490. [CrossRef]

53. Hakki, S.S.; Tatar, G.; Dundar, N.; Demiralp, B. The effect of different cleaning methods on the surface and temperature of failed titanium implants: An in vitro study. Lasers Med. Sci. 2017, 32, 563-571. [CrossRef]

54. Kister, F.; Specht, O.; Warkentin, M.; Geis-Gerstorfer, J.; Rupp, F. Peri-implantitis cleaning instrumentation influences the integrity of photoactive nanocoatings. Dent. Mater. 2017, 33, e69-e78. [CrossRef] [PubMed]

55. Ronay, V.; Merlini, A.; Attin, T.; Schmidlin, P.R.; Sahrmann, P. In vitro cleaning potential of three implant debridement methods. Simulation of the non-surgical approach. Clin. Oral Implant. Res. 2017, 28, 151-155. [CrossRef] [PubMed]

56. Bertoldi, C.; Lusuardi, D.; Battarra, F.; Sassatelli, P.; Spinato, S.; Zaffe, D. The maintenance of inserted titanium implants: In-vitro evaluation of exposed surfaces cleaned with three different instruments. Clin. Oral Implant. Res. 2017, 28, 57-63. [CrossRef]

57. Larsen, O.I.; Enersen, M.; Kristoffersen, A.K.; Wennerberg, A.; Bunæs, D.F.; Lie, S.A.; Leknes, K.N. Antimicrobial Effects of Three Different Treatment Modalities on Dental Implant Surfaces. J. Oral Implantol. 2017, 43, 429-436. [CrossRef]

58. Tastepe, C.S.; Lin, X.; Donnet, M.; Wismeijer, D.; Liu, Y. Parameters That Improve Cleaning Efficiency of Subgingival Air Polishing on Titanium Implant Surfaces: An In Vitro Study. J. Periodontol. 2017, 88, 407-414. [CrossRef]

59. Chen, C.J.; Ding, S.J.; Chen, C.C. Effects of Surface Conditions of Titanium Dental Implants on Bacterial Adhesion. Photomed. Laser Surg. 2016, 34, 379-388. [CrossRef]

60. Rios, F.G.; Viana, E.R.; Ribeiro, G.M.; González, J.C.; Abelenda, A.; Peruzzo, D.C. Temperature evaluation of dental implant surface irradiated with high-power diode laser. Lasers Med. Sci. 2016, 31, 1309-1316. [CrossRef]

61. Park, J.B.; Lee, S.H.; Kim, N.; Park, S.; Jin, S.H.; Choi, B.K.; Kim, K.K.; Ko, Y. Instrumentation with Ultrasonic Scalers Facilitates Cleaning of the Sandblasted and Acid-Etched Titanium Implants. J. Oral Implantol. 2015, 41, 419-428. [CrossRef]

62. Anastassiadis, P.M.; Hall, C.; Marino, V.; Bartold, P.M. Surface scratch assessment of titanium implant abutments and cementum following instrumentation with metal curettes. Clin. Oral Investig. 2015, 19, 545-551. [CrossRef]

63. Sahrmann, P.; Ronay, V.; Hofer, D.; Attin, T.; Jung, R.E.; Schmidlin, P.R. In vitro cleaning potential of three different implant debridement methods. Clin. Oral Implant. Res. 2015, 26, 314-319. [CrossRef] [PubMed]

64. Park, J.B.; Jang, Y.J.; Choi, B.K.; Kim, K.K.; Ko, Y. Treatment with various ultrasonic scaler tips affects efficiency of brushing of SLA titanium discs. J. Craniofac. Surg. 2013, 24, e119-e123. [CrossRef] [PubMed]

65. Sahrmann, P.; Ronay, V.; Sener, B.; Jung, R.E.; Attin, T.; Schmidlin, P.R. Cleaning potential of glycine air-flow application in an in vitro peri-implantitis model. Clin. Oral Implant. Res. 2013, 24, 666-670. [CrossRef] [PubMed]

66. Mussano, F.; Rovasio, S.; Schierano, G.; Baldi, I.; Carossa, S. The effect of glycine-powder airflow and hand instrumentation on peri-implant soft tissues: A split-mouth pilot study. Int. J. Prosthodont. 2013, 26, 42-44. [CrossRef]

67. Vieira, L.F.N.; Cardoso, E.S.; Machado, S.J.; da Silva, C.P.; Vidigal, G.M., Jr. Effectiveness of implant surface decontamination using a high-pressure sodium bicarbonate protocol: An in vitro study. Implant. Dent. 2012, 21, 390-393. [CrossRef] [PubMed]

68. Park, J.B.; Kim, N.; Ko, Y. Effects of ultrasonic scaler tips and toothbrush on titanium disc surfaces evaluated with confocal microscopy. J. Craniofac. Surg. 2012, 23, 1552-1558. [CrossRef] [PubMed]

69. Schmage, P.; Thielemann, J.; Nergiz, I.; Scorziello, T.M.; Pfeiffer, P. Effects of 10 cleaning instruments on four different implant surfaces. Int. J. Oral Maxillofac. Implant. 2012, 27, 308-317. 